Universidade Tecnológica Federal do Paraná - UTFPR

Campus Ponta Grossa - Paraná - Brasil

ISSN: $1981-3686 /$ v. 04, n. 02 : p. $218-234,2010$

DOI: $10.3895 / \mathrm{S} 1981-36862010000200010$
Revista Brasileira de Tecnologia

Agroindustrial

\title{
INFLUÊNCIA DE PRÉ-TRATAMENTOS NA CINÉTICA DE SECAGEM EM DIFERENTES TEMPERATURAS DE BANANA DA VARIEDADE TERRA (Musa Sapientum, Linneo)
}

\section{INFLUENCE OF PRETREATMENTS ON THE KINETICS OF DRYNG AT DIFFERENT TEMPERATURES OF BANANA VARIETY TERRA (Musa sapientum, Linneo)}

\author{
Philipe dos Santos ${ }^{1}$, Fabrício Schwanz da Silva ${ }^{2}$, Alexandre Gonçalves Porto ${ }^{3}$, \\ Flávio Teles Carvalho da Silva ${ }^{4}$ Guilherme de Figueiredo Furtado ${ }^{5}$. \\ ${ }^{1}$ Universidade do Estado de Mato Grosso - UNEMAT - Barra do Bugres ph sinop@ hotmail.com \\ ${ }^{2}$ Universidade do Estado de Mato Grosso - UNEMAT - Barra do Bugres fabricio@ unemat.br \\ ${ }^{3}$ Universidade do Estado de Mato Grosso - UNEMAT - Barra do Bugres agporto@ unemat.br \\ ${ }^{4}$ Universidade do Estado de Mato Grosso - UNEMAT - Barra do Bugres ftcsilva@ gmail.com \\ ${ }^{5}$ Universidade do Estado de Mato Grosso - UNEMAT - Barra do Bugresguilherme.sartori@ hotmail.com
}

\begin{abstract}
Resumo
O presente trabalho foi conduzido com o objetivo de estudar a influência de diferentes prétratamentos na cinética de secagem de banana da variedade Terra. Os frutos de banana foram submetidos a três pré-tratamentos: espessura da fatia $(2$ e $4 \mathrm{~mm}$ ) branqueamento (sim e não) $e$ concentração de $\mathrm{NaCl}(2$ e $5 \%$ ) na desidratação osmótica. As secagens foram realizadas utilizando-se um secador descontínuo de bandejas em escala de bancada, com temperaturas de 60 e $80{ }^{\circ} \mathrm{C}$ e velocidade de $1 \mathrm{~m} . \mathrm{s}^{-1}$. Quatro diferentes modelos empíricos foram ajustados aos dados experimentais de umidade e comparados. Para analisar os efeitos dos pré-tratamentos na cinética secagem de banana da variedade terra, utilizou-se um planejamento experimental fracionário $2_{I I I}^{4-}$ ${ }^{1}$, com quatro fatores e dois níveis de variação, para a constante de secagem (K). Dentre os modelos testados o Modelo de Midilli e Kucuk apresentou os maiores coeficiente de determinação $\left(R^{2}\right)$ e os menores erros médios estimados (SE), sendo o mesmo utilizado para a determinação da constante de secagem. O modelo matemático de Midilli e Kucuk foi o que melhor ajustou-se aos dados experimentais; a espessura das amostras e a temperatura de operação do secador influenciaram significativamente $(p=0,05)$ a constante de secagem, sendo diretamente proporcional ao aumento da temperatura de operação do secador e inversamente proporcional à espessura das amostras. De acordo com as constantes de secagem apresentadas pelo modelo, recomenda-se a secagem de banana da variedade terra nas seguintes condições: espessura de $2 \mathrm{~mm}$, temperatura de secagem $80{ }^{\circ} \mathrm{C}$ e qualquer outro pré-tratamento, desidratação osmótica com $\mathrm{NaCl}$ elou branqueamento.
\end{abstract}

Palavras-chave: banana da terra; constante de secagem; planejamento experimental. 


\section{Introdução}

As frutas tropicais têm evoluído bastante na preferência dos consumidores mais exigentes, principalmente, no que se refere às características de sabor e aroma, existindo uma tendência de crescimento no mercado nacional (GURJÃO, 2006).

A banana (Musa spp.), é originária do Continente Asiático e, atualmente, vem sendo explorada na maioria dos países tropicais (MATTHIESEN e BOTEON, 2003). Esta fruta é um componente constante na dieta dos brasileiros, apenas um fruto de banana pode conter um valor energético de 380kJ, além de fornecer quantidades significativas de vitaminas A e B, potássio e outros minerais, como o sódio (JESUS et al., 2004). Segundo Souza Neto et al. (2005) a banana da variedade terra possui potencial de ser utilizada como fruta desidratada e necessita de novos estudos para conhecer o seu comportamento perante os tratamentos e processos da operação de secagem.

Um dos principais problemas desta fruta é a sua alta perecividade, por isto o desenvolvimento de técnicas para conservação se torna de extrema importância. Dentre as técnicas empregadas para conservação pós-colheita de produtos agrícolas, a desidratação se destaca por proporcionar uma maior estabilidade, diminuir a degradação enzimática e oxidativa, reduzir custos com transporte e pela disponibilidade do produto em qualquer época do ano (PARK et al., 2001; FELLOWS, 1994).

As operações de desidratação e/ou secagem, envolvem a transferência simultânea de calor e massa, no qual o calor é transferido para o produto e a umidade é removida na forma de vapor d'água para uma fase gasosa insaturada (VASQUES et al., 2004). Strumilo e Kudra (1986) afirmam que na secagem, o material úmido entra em contato com ar insaturado resultando uma diminuição da umidade do material e a umidificação do ar. Nesse processo, o calor é requerido para evaporar a umidade durante a secagem do produto e a água evaporada na superfície externa do produto é removida e transferida para o meio de secagem que, normalmente, é o ar (BROOKER et al., 1992; GEANKOPLIS, 1998).

A taxa de secagem de frutos e vegetais, pode ser aumentada pela remoção da resistência da superfície ao transporte de água, através do uso de tratamentos químicos ou físicos (SILVA et al., 2003). Dentre os pré-tratamentos existentes, a espessura de fatia, branqueamento e a desidratação osmótica destacam-se por serem utilizados, geralmente, para aumentar a taxa de secagem, prevenir sabores indesejáveis e mudanças na coloração dos produtos, resultante de reações enzimáticas (SILVA et al., 2008).

O branqueamento tem sido um dos mais populares métodos de prevenção do escurecimento enzimático (CAMARGO, 1986). Segundo Dandamrongrak et al. (2002), o branqueamento é um 
importante passo no processamento de frutas e hortaliças, em que sua aplicação inibe a ação enzimática e aumenta a taxa de secagem.

A desidratação osmótica é uma técnica que consiste na imersão da matéria prima em soluções aquosas concentradas de açúcares ou sais, levando a dois fluxos de massa simultâneos: um fluxo de água do alimento para a solução e uma transferência simultânea de soluto da solução para o alimento (ANDRADE et al., 2003; MARQUES et al. 2007). Segundo Marques et al. (2007) a desidratação osmótica juntamente com a secagem convectiva é um processo que permite a obtenção de frutas com melhor estabilidade, cor, textura e aumento da vida de prateleira.

Diversos modelos teóricos, semi-teóricos e empíricos, têm sido empregados para explicar o fenômeno de secagem. Estes são utilizados para se estimar o tempo de secagem de diferentes produtos e gerar as curvas de secagem (MIDILLI et al., 2002). Entre os modelos semi-teóricos e empíricos, Aproximação da Difusão, Dois Termos, Midilli e Kucuk e Page, tem sido frequientemente utilizados para a modelagem matemática da cinética de secagem de produtos agrícolas (CORREAA et al., 2007; DOYMAZ, 2007b; MIDILLI et al., 2002; PANCHARIYA et al., 2002; OZDEMIR e DEVRES, 1999).

A difusividade efetiva de umidade é uma importante propriedade de transporte, sendo útil na análise das operações de processamento de produtos alimentícios. Porém, devido à complexa composição e a estrutura física dos alimentos, não são conseguidas estimativas precisas desta propriedade, sendo assim necessárias medidas experimentais (SARAVACOS e ROUZEOUS, 1984). Pode-se entender a difusividade como a facilidade com que a água é removida do material (BROD, 2003).

Devido ao exposto acima, o presente trabalho teve como objetivos, submeter à fruta a diferentes pré-tratamentos, e com isso caracterizar a secagem da banana da terra (Musa sapientum, Linneo), ajustar os diferentes modelos matemáticos as curvas de secagem, determinar as constantes de secagem (K) e a difusividade efetiva de umidade, bem como utilizar a metodologia do planejamento experimental fatorial na análise estatística da secagem de banana da variedade terra.

\section{Material e Métodos}

O presente trabalho foi desenvolvido no Laboratório de Engenharia e Processamento Agroindustrial, vinculado ao Centro de Tecnolgia do Mato Grosso (CTMAT), localizados no Campus Universtário “Dep. Estadual Renê Barbour”, da Universidade do Estado de Mato Grosso, localizada no município de Barra do Bugres-MT.

A matéria prima utilizada foi a banana da variedade terra (Musa sapientum, Linneo) produzidas e comercializadas na região do médio norte mato-grossense. As frutas selecionadas para 
o processo de secagem apresentavam-se amareladas com pintas pretas e no estádio de maturação madura, com aproximadamente $23^{\circ}$ Brix conforme descrito por Pontes et al., 2007.

As frutas selecionadas foram descascadas e posteriormente submetidas a três prétratamentos: espessura da fatia $(2$ e $4 \mathrm{~mm})$ fatiadas em um multiprocessador PHILIPS WALITA MOD. RI 7743 e branqueamento (sim e não) em água fervente $\left(100^{\circ} \mathrm{C}\right)$ durante 3 minutos e resfriadas com imersão em água aproximadamente $8^{\circ} \mathrm{C}$. Finalmente as fatias foram desidratadas osmóticamente em duas concentrações de cloreto de sódio, 2 e $5 \%$, durate 30 minutos a $26 \pm 2{ }^{\circ} \mathrm{C}$. O excesso de água nas amostras, poveniente dos processos anteriores, foi retirado com o auxilio de peneiras, por fim, as amostras foram transferidas para o secador de bandejas.

As secagens foram realizadas utilizando-se um secador descontínuo de bandejas em escala de bancada (Figura 1), conforme o modelo de secador descrito por Porto (2001). As amostras foram colocadas em bandejas inoxidáveis de fundo maciço, com $11 \mathrm{~cm}$ de comprimento por $11 \mathrm{~cm}$ de largura e $4 \mathrm{~mm}$ de espessura, e inseridas na câmara de secagem. As temperaturas do ar de secagem foram de $60^{\circ}$ e $80^{\circ} \mathrm{C}$ e a velocidade do ar foi de aproximadamente $1 \mathrm{~m} . \mathrm{s}^{-1}$, garantindo que a resistência à transferência de massa seja controlada pela migração de água no interior do material.

Figura 1 - Secador descontínuo de bandejas em escala de bancada.

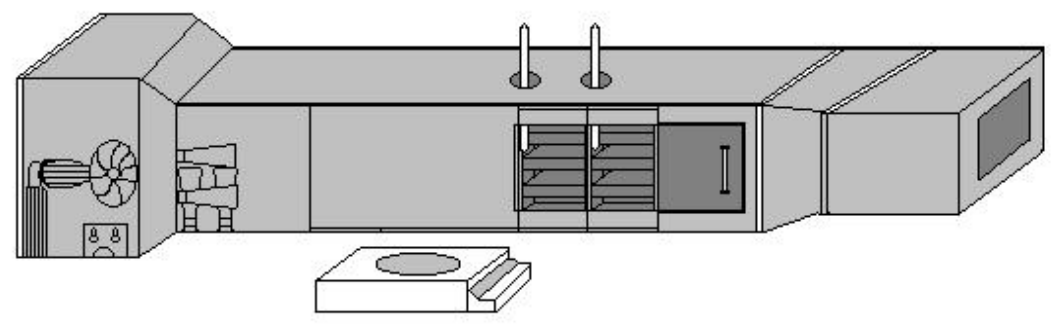

Fonte: PORTO (2001)

As amostras foram retiradas do equipamento para a realização das medidas das massas em balança semi-analítica OHAUS Modelo AR3130 com precisão de 0,001g. A verificação ocorreu em intervalos de 15 em 15 minutos, até peso constante. As amostras permaneceram no secador até completar o 24 horas, realizando-se, então, o último registro de massa, para a obtenção da umidade de equilíbrio. As amostras foram replicadas para uma melhor confiabilidade dos resultados.

A razão de umidade (RU), adminesional, durante a secagem nas diferentes condições, foi determinada por meio da seguinte expressão:

$$
\mathrm{RU}=\frac{\mathrm{U}^{*}-\mathrm{U}^{*} \mathrm{e}}{\mathrm{U}_{\mathrm{i}}^{*}-\mathrm{U}^{*} \mathrm{e}}
$$


onde: $\mathrm{RU}=$ razão de umidade do produto, adimensional; $\mathrm{U}_{\mathrm{e}}{ }_{\mathrm{e}}=$ teor de água de equilíbrio do produto, decimal (b.s.); $\mathrm{U}^{*}{ }_{\mathrm{i}}=$ teor de água inicial do produto, decimal (b.s.); $\mathrm{U}^{*}=$ teor de água do produto, decimal (b.s.).

Modelos matemáticos empíricos utilizados por vários pesquisadores para predizer o fenômeno da secagem de produtos agrícolas foram ajustados aos dados observados de razão de umidade (AFONSO JÚNIOR e CORRÊA, 1999; AKPINAR et al., 2003; ERTEKIN e YALDIZ, 2004; LAHSASNI et al., 2004). Na Tabela 1, apresentam-se os modelos que foram avaliados neste trabalho.

Tabela 1 - Modelos matemáticos utilizados para predizer o fenômeno de secagem.

\begin{tabular}{lcc}
\hline Designação do Modelo & Modelo & Referência \\
\hline Aproximação da Difusão & $\mathrm{RU}=\mathrm{a} \exp (-\mathrm{kt})+(1-\mathrm{a}) \exp (-\mathrm{kbt})$ & MENGES e ERTEKIN (2006) \\
Dois Termos & $\mathrm{RU}=\mathrm{a} \exp \left(-\mathrm{k}_{\mathrm{o}} \mathrm{t}\right)+\mathrm{b} \exp \left(-\mathrm{k}_{1} \mathrm{t}\right)$ & DANDAMRONGRAK et al. \\
Midilli e Kucuk & $\mathrm{RU}=\mathrm{a} \exp \left(-\mathrm{kt}^{\mathrm{n}}\right)+\mathrm{bt}$ & MIDILLI et al. (2002) \\
Page & $\mathrm{RU}=\exp \left(-\mathrm{kt}^{\mathrm{n}}\right)$ & GONELI et al. (2009)
\end{tabular}

Em que: RU - razão de umidade do produto, adimensional; $\mathrm{t}$ - tempo de secagem, $\mathrm{s}, \mathrm{h} ; \mathrm{k}, \mathrm{k}_{0}, \mathrm{k}_{1}$-coeficientes de secagem, $\mathrm{h}^{-1} ; \mathrm{a}, \mathrm{b}, \mathrm{c}, \mathrm{n}$ - constantes dos modelos. Fonte: Autores 2010

Para determinação da difusividade efetiva $\left(\mathrm{D}_{\mathrm{EF}}\right)$, utilizou-se o modelo difusivo de Fick, que despreza o encolhimento para o perfil médio de umidade (lâmina infinita, secagem por um lado), representado na seguinte expressão:

$$
R U=\left(\frac{8}{\pi^{2}}\right) * \sum_{n=0}^{\infty} \frac{1}{(2 n+1)^{2}} \exp \left[-(2 n+1)^{2} * \pi^{2} * \frac{D_{E F} * t}{4 * L_{0}^{2}}\right]
$$

em que: $\mathrm{L}_{0}$ - espessura da camada de produto.

Para o ajuste dos modelos, foram realizadas análises de regressão não linear, pelo método Gauss-Newton. O grau de ajuste de cada modelo considerou a magnitude do coeficiente de determinação $\left(\mathrm{R}^{2}\right)$ e o erro médio estimado (SE).

$$
S E=\sqrt{\frac{\sum\left(Y-Y_{0}\right)^{2}}{G L R}}
$$


em que: $\mathrm{Y}$ - valor observado experimentalmente; $\mathrm{Y}_{0}$ - valor calculado pelo modelo; GLR graus de liberdade do modelo.

Para analisar o efeito dos pré-tratamentos (espessura, branqueamento e concentração de cloreto de sódio na desidratação osmótica) e da temperatura de secagem de banana da terra, utilizou-se o um planejamento experimental fracionário $2_{\mathrm{III}}^{4-1}$, com quatro fatores (X) em analise e dois níveis de variação, para o fator de resposta constante de secagem (K). Na matriz de planejamento experimental, os valores das variáveis estão representados na forma codificada ( -1 e +1 ), conforme Rodrigues e Iemma (2005), sendo os fatores e os respectivos níveis apresentados na Tabela 2.

Tabela 2 - Variáveis e níveis utilizados no planejamento experimental fatorial fracionário $\left(2_{\text {III }}^{4-1}\right)$

\begin{tabular}{ccc}
\hline Fatores & \multicolumn{2}{c}{ Níveis } \\
\cline { 2 - 3 } & $\mathbf{- 1}$ & $\mathbf{+ 1}$ \\
\hline Espessura & $2 \mathrm{~mm}$ & $4 \mathrm{~mm}$ \\
Branqueamento & $\mathrm{Não}$ & Sim \\
Concentração de $\mathrm{NaCl}$ & $2 \%$ & $5 \%$ \\
Temperatura & $60^{\circ} \mathrm{C}$ & $80^{\circ} \mathrm{C}$ \\
\hline
\end{tabular}

Fonte: Autores 2010

A matriz do planejamento experimental fracionário que foi utilizada para a secagem de bananas da terra, com réplicas, tendo como resposta a constante de secagem (K), é mostrada na Tabela 3.

Tabela 3 - Matriz de planejamento experimental fatorial fracionário $\left(2_{\mathrm{III}}^{4-1}\right)$ para a constante de secagem $(\mathrm{K})$.

\begin{tabular}{ccccc}
\hline SECAGEM & $\mathbf{X}_{\text {Espessura }}$ & $\mathbf{X}_{\text {Branqueamento }}$ & $\mathbf{X}_{\text {Concentracão de NaCl }}$ & $\mathbf{X}_{\text {Temperatura }}$ \\
\hline 1 & -1 & -1 & -1 & -1 \\
2 & +1 & -1 & -1 & +1 \\
3 & -1 & +1 & -1 & +1 \\
4 & +1 & +1 & -1 & -1 \\
5 & -1 & -1 & +1 & +1 \\
6 & +1 & -1 & +1 & -1 \\
7 & -1 & +1 & +1 & -1 \\
8 & +1 & +1 & +1 & +1 \\
\hline
\end{tabular}

Fonte: Autores 2010

O planejamento experimental utilizado, Tabela 3, foi analisado através do software Mitab 15 e a superfície de resposta gerada no software MatLab 7.0, estabelecendo assim, a influência dos prétratamentos: espessura de fatia (2 e $4 \mathrm{~mm}$ ), branqueamento (sim ou não), concentração osmótica (2 e $5 \%)$ e também a variável de processo temperatura de secagem $\left(60^{\circ}\right.$ e $\left.80^{\circ} \mathrm{C}\right)$ em relação as constantes de secagem $(\mathrm{K})$, geradas pelo melhor modelo ajustado as dados experimentais. 


\section{Resultados e Discussão}

Nas análises dos dados de secagem, a razão de umidade é essencial para descrever diferentes modelos de secagem. A cada período de secagem, um teor de água é correlacionado com o teor de água inicial e o teor de água de equilíbrio, para condições especificas de secagem. Desta forma, em todas as condições de secagem testadas, os diferentes modelos matemáticos utilizados para descrever a cinética de secagem foram ajustados aos valores da razão de umidade em função do tempo de secagem.

Apresentam-se, nas Figuras 2 e 3, as curvas de secagem para os experimentos realizados da matriz experimental e as réplicas, respectivamente.

Figura 2 - Curva da razão de umidade (RU) em função do tempo para as secagens da matriz experimental.

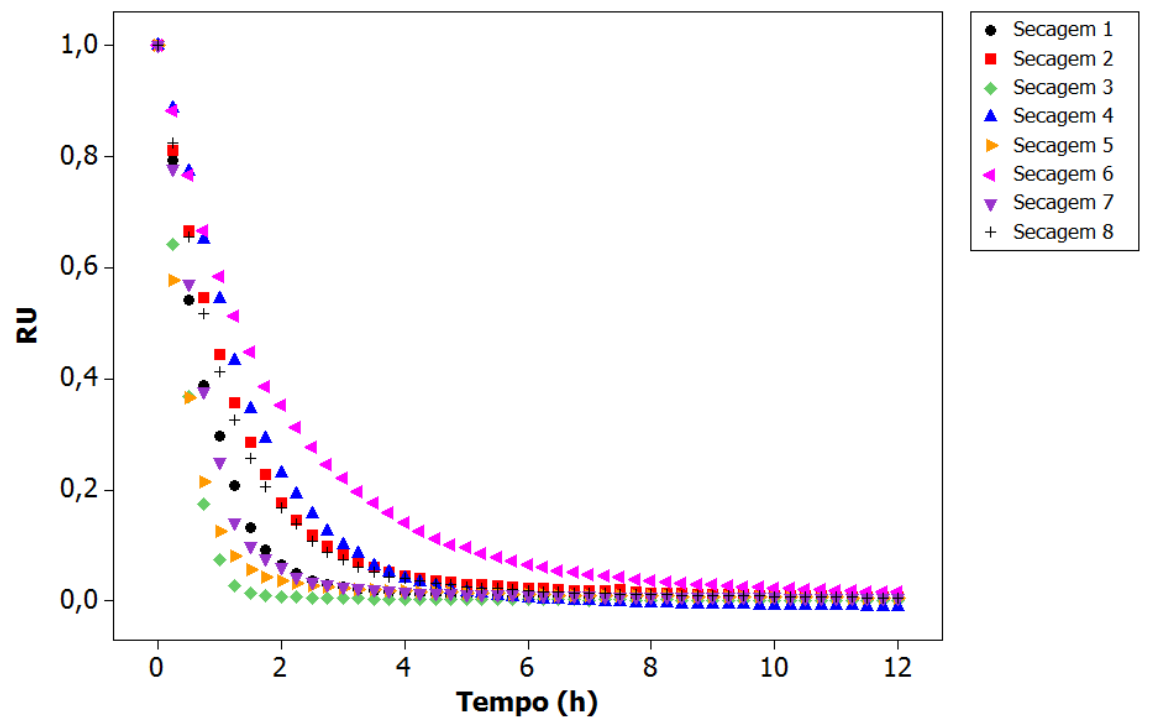

Figura 3 - Curva da razão de umidade (RU) em função do tempo para as réplicas das secagens da matriz experimental

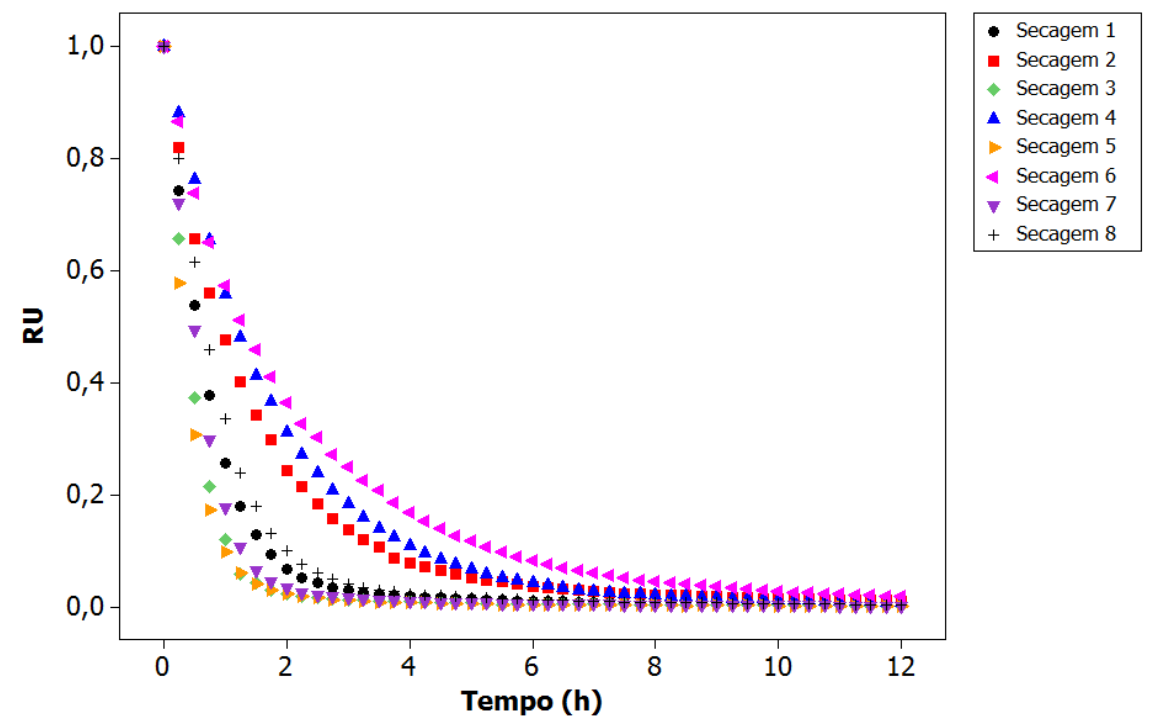

Analisando as curvas de razão de umidade, demonstradas nas Figuras 2 e 3, verifica-se que o 
aumento da temperatura favorece o processo de transferência de energia na forma calor para as amostras, conseqüentemente diminuindo o tempo necessário para a fruta atingir o equilíbrio, o mesmo comportamento foi observado por Kaleemullah e Kailappan (2006), Doymaz (2005) e Wang et al. (2007).

$\mathrm{Na}$ Tabela 4 são apresentados os valores dos coeficientes de determinação $\left(\mathrm{R}^{2}\right.$, em \%) e o erro médio estimado (SE, em decimal) para os quatro modelos analisados para as secagens da matriz experimental, com as suas respectivas réplicas.

Tabela 4 - Valores do coeficiente de determinação $\left(\mathrm{R}^{2}\right)$ e do erro médio estimado (SE), calculados para verificação do ajuste dos modelos matemáticos aos valores experimentais da secagem para as secagens da matriz experimental, com as suas respectivas réplicas .

\begin{tabular}{|c|c|c|c|c|c|c|c|c|}
\hline \multirow{2}{*}{ SECAGEM } & \multicolumn{2}{|c|}{$\begin{array}{l}\text { Aproximação da } \\
\text { Difusão }\end{array}$} & \multicolumn{2}{|c|}{ Dois Termos } & \multicolumn{2}{|c|}{ Midilli e Kucuk } & \multicolumn{2}{|c|}{ Page } \\
\hline & $\mathbf{R}^{2}(\%)$ & SE & $\mathbf{R}^{2}(\%)$ & SE & $\mathbf{R}^{2}(\%)$ & SE & $\mathbf{R}^{2}(\%)$ & SE \\
\hline 1 & 99,87 & 0,010088 & 99,86 & 0,007595 & 99,93 & 0,010805 & 99,90 & 0,008666 \\
\hline 2 & 99,95 & 0,006767 & 99,86 & 0,006788 & 99,95 & 0,01185 & 99,86 & 0,011454 \\
\hline 3 & 99,71 & 0,013311 & 99,72 & 0,003594 & 99,98 & 0,01353 & 99,97 & 0,004011 \\
\hline 4 & 99,83 & 0,013311 & 99,79 & 0,003594 & 99,98 & 0,01353 & 99,96 & 0,004011 \\
\hline 5 & 99,83 & 0,009895 & 99,70 & 0,008742 & 99,87 & 0,013369 & 99,75 & 0,011869 \\
\hline 6 & 99,95 & 0,007799 & 99,81 & 0,004527 & 99,98 & 0,015041 & 99,92 & 0,009682 \\
\hline 7 & 99,72 & 0,015084 & 99,67 & 0,009537 & 99,89 & 0,016532 & 99,82 & 0,011961 \\
\hline 8 & 99,95 & 0,006623 & 99,89 & 0,006347 & 99,96 & 0,01032 & 99,89 & 0,010182 \\
\hline 1 , & 99,91 & 0,008164 & 99,86 & 0,010492 & 99,93 & 0,007429 & 99,86 & 0,010146 \\
\hline 2 ' & 99,83 & 0,012997 & 99,97 & 0,005081 & 99,98 & 0,004452 & 99,91 & 0,009401 \\
\hline $3^{\prime}$ & 99,86 & 0,00919 & 99,83 & 0,010381 & 99,93 & 0,006531 & 99,89 & 0,008085 \\
\hline $4^{\prime}$ & 99,98 & 0,004827 & 99,92 & 0,009464 & 99,98 & 0,004499 & 99,94 & 0,008264 \\
\hline 5, & 99,93 & 0,00602 & 99,98 & 0,003488 & 99,93 & 0,006081 & 99,91 & 0,00716 \\
\hline 6 ' & 99,74 & 0,017292 & 99,66 & 0,020076 & 99,99 & 0,003327 & 99,97 & 0,005717 \\
\hline 7 ' & 99,80 & 0,011923 & 99,78 & 0,012754 & 99,93 & 0,006863 & 99,90 & 0,008284 \\
\hline $8^{\prime}$ & 99,89 & 0,009849 & 99,83 & 0,012182 & 99,93 & 0,008029 & 99,86 & 0,011093 \\
\hline
\end{tabular}

Fonte: Autores 2010

Conforme pode ser observado na tabela acima, verifica-se que todos os modelos analisados apresentaram ajustes com elevados coeficientes de determinação $\left(\mathrm{R}^{2}\right)$, superiores ou próximas a 99\%, e baixo erro médio estimado inferiores a 0,020, segundo Goneli et al. (2009) tais resultados indicam uma representação satisfatória do processo de secagem.

O modelo de Midilli e Kucuk foi o que melhor se ajustou aos dados observados, nas diferentes condições de secagem, pois apresentou o melhor coeficiente de determinação $\left(\mathrm{R}^{2}\right)$ e o menor erro médio estimado (SE). Menges e Ertekin (2006) ao analisarem a cinética de secagem de maçãs douradas verificaram que o modelo de Midilli e Kucuk ajustou-se com elevados valores de coeficientes de determinação e baixo erro médio estimado.

Na Figura 4 é apresentado, para todas as condições de secagem estudadas, o gráfico de correspondência entre os teores de água observados e os estimados pelo modelo de Midilli e Kucuk para as secagens da matriz experimental, com as suas respectivas réplicas. 
Figura 4 - Valores de correspondência entre valores estimados e observados pelo modelo de Midilli e Kucuk para as secagens da matriz experimental, com as suas respectivas réplicas.

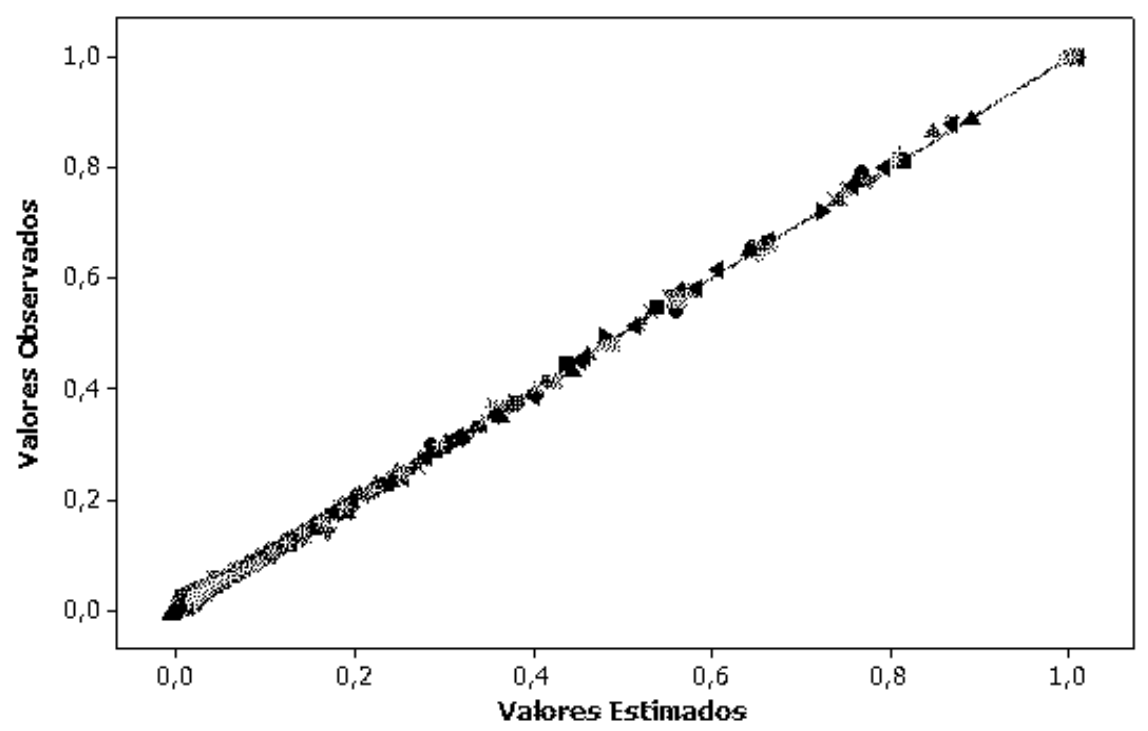

Os resultados apresentados na Figura 4 permitem confirmar o excelente ajuste do modelo de Midilli e Kucuk aos dados observados, descrevendo adequadamente a cinética de secagem da banana da variedade terra, resultado semelhante foi encontrado em trabalhos de Wang et al. (2007), Menges e Ertelin (2006) e Sacilik, et al. (2006), estudando a cinética de secagem de maçã, maçã variedade ouro e tomate orgânico, respectivamente.

Os valores dos parâmetros estimados pelo ajuste do modelo de Midilli e Kucuk e a difusividade efetiva $\left(\mathrm{D}_{\mathrm{EF}}\right)$ estão apresentados na Tabela 5.

Tabela 5 - Parâmetros obtidos do modelo de Midilli e Kucuk e difusividade efetiva $\left(\mathrm{D}_{\mathrm{EF}}\right)$ ajustados aos dados de secagem da matriz experimental, com as suas respectivas réplicas.

\begin{tabular}{cccccc}
\hline SECAGEM & $\mathbf{a}$ & $\mathbf{K}$ & $\mathbf{n}$ & $\mathbf{b}$ & $\mathbf{D}_{\mathbf{E F}}\left(\mathbf{m}^{2} \cdot \mathbf{s}^{-1}\right)$ \\
\hline 1 & 1,008019 & 1,262511 & 1,103464 & 0,000605 & $5,68529 \times 10^{-1}$ \\
2 & 1,001816 & 0,832965 & 1,006294 & 0,001391 & $3,75098 \times 10^{-10}$ \\
3 & 0,998152 & 2,507277 & 1,276327 & 0,000269 & $1,12907 \times 10^{-9}$ \\
4 & 1,006740 & 0,631379 & 1,177484 & $-0,000573$ & $2,8432 \times 10^{-10}$ \\
5 & 1,001732 & 1,959030 & 0,911124 & 0,001165 & $8,82183 \times 10^{-10}$ \\
6 & 1,011239 & 0,552403 & 0,933719 & 0,001531 & $2,48756 \times 10^{-10}$ \\
7 & 1,004144 & 1,373296 & 1,206787 & 0,001054 & $6,18418 \times 10^{-10}$ \\
8 & 1,009964 & 0,891998 & 1,003617 & 0,001154 & $4,01681 \times 10^{-10}$ \\
1 & 1,004758 & 1,318075 & 1,042838 & 0,001001 & $5,93551 \times 10^{-10}$ \\
2 & 1,005048 & 0,755228 & 0,883174 & 0,001373 & $3,40092 \times 10^{-10}$ \\
3 & 1,002936 & 2,110969 & 1,129739 & 0,000695 & $9,50604 \times 10^{-10}$ \\
4 & 1,011805 & 0,588437 & 0,978375 & 0,001090 & $2,64983 \times 10^{-10}$ \\
5 & 1,002600 & 2,277327 & 0,994894 & 0,000559 & $1,02552 \times 10^{-9}$ \\
6 & 1,006795 & 0,555662 & 0,852035 & 0,000947 & $2,50224 \times 10^{-10}$ \\
7 & 1,001146 & 1,669269 & 1,180321 & 0,000684 & $7,51699 \times 10^{-10}$ \\
8, & 1,007213 & 1,082872 & 1,087006 & 0,001105 & $4,87635 \times 10^{-10}$ \\
\hline
\end{tabular}

Analisando os resultados obtidos (Tabela 5), observa-se que a magnitude da constante de 
secagem (K) para o modelo de Midilli e Kucuk, que representa o efeito das condições externas de secagem, aumentou com a elevação da temperatura do ar de secagem. Resultado semelhante foi encontrado por Menges e Ertekin (2006), Wang et al. (2007), Park et al. (2001) e Akpinar et al.(2003) ao estudarem a secagem de maçã dourada, polpa de maçã, pêra e pimentão, respectivamente. Segundo Babalis e Belessiotis (2004), a constante de secagem (K) pode ser utilizada como aproximação para caracterizar o efeito da temperatura e está relacionada à difusividade efetiva de umidade no processo de secagem.

Os valores da difusividade efetiva $\left(\mathrm{D}_{\mathrm{EF}}\right)$ para as secagens da matriz de planejamento experimental variaram entre 2,48756 x $10^{-10}$ a $1,02552 \times 10^{-9} \mathrm{~m}^{2} \cdot \mathrm{s}^{-1}$. Segundo Zogzas et al. (1996), os valores de difusividade efetiva $\left(\mathrm{D}_{\mathrm{EF}}\right)$ para produtos de origem agrícola se encontram na faixa de $10^{-11}$ a $10^{-9} \mathrm{~m}^{2} \mathrm{~s}^{-1}$.

Verifica-se, a partir da Tabela 5, que os valores de difusividade efetiva $\left(\mathrm{D}_{\mathrm{EF}}\right)$, aumentaram com a elevação da constante de secagem $(\mathrm{K})$, demonstrando a diminuição das resistências internas de secagem. O mesmo comportamento foi observado em outros estudos de secagem de produtos de origem vegetal: destacando-se os trabalhos de Park et al. (2001), Doymaz (2007a), Goyal et al. (2006) e Sacilik e Konuralp (2006), secando pêra, tomate, manga e maçã de cultivo orgânico, respectivamente. Nguyen e Price (2007) estudaram a secagem de banana nas temperaturas de 50, 60 e $70^{\circ} \mathrm{C}$ e encontraram para a difusividade efetiva $\left(D_{E F}\right)$ valores entre 3,2 a $7,8 \times 10^{-10} \mathrm{~m}^{2} \cdot \mathrm{s}^{-1}$, para amostras fatiadas a $10 \mathrm{~mm}$ de espessura e de 10,8 a $22,7 \times 10^{-10} \mathrm{~m}^{2} \cdot \mathrm{s}^{-1}$ para as amostras com $20 \mathrm{~mm}$.

Para estudar as influências dos pré-tratamentos utilizados na operação de secagem bem como a temperatura de secagem realizou-se um planejamento experimental do tipo fatorial fracionário $2_{\text {III }}^{4-1}$ em dois níveis de variação com quatro fatores, tendo como resposta a constante de secagem (K). A Tabela 6 apresenta a matriz do planejamento com as constantes de secagem (K) obtidas pelo modelo matemático ajustado aos dados experimentais.

Tabela 6 - Matriz de planejamento experimental fatorial fracionário $2_{\text {III }}^{4-1}$ para a constante de secagem $(\mathrm{K})$.

\begin{tabular}{cccccc}
\hline SECAGEM & $\mathbf{X}_{\text {Espessura }}$ & $\mathbf{X}_{\text {Branqueamento }}$ & $\mathbf{X}_{\text {Concentracão de NaCl }}$ & $\mathbf{X}_{\text {Temperatura }}$ & $\mathbf{K}_{\left(\mathbf{m i n}^{-1}\right)}$ \\
\hline 1 & -1 & -1 & -1 & -1 & 1,262511 \\
2 & +1 & -1 & -1 & +1 & 0,832965 \\
3 & -1 & +1 & -1 & +1 & 2,507277 \\
4 & +1 & +1 & -1 & -1 & 0,631379 \\
5 & -1 & -1 & +1 & +1 & 1,959030 \\
6 & +1 & -1 & +1 & -1 & 0,552403 \\
7 & -1 & +1 & +1 & -1 & 1,373296 \\
8 & +1 & +1 & -1 & +1 & 0,891998 \\
1, & -1 & -1 & -1 & -1 & 1,318075 \\
2, & +1 & -1 & -1 & +1 & 0,755228 \\
3, & -1 & +1 & -1 & +1 & 2,110969 \\
4 & +1 & +1 & +1 & -1 & 0,588437 \\
5, & -1 & -1 & +1 & +1 & 2,277327 \\
6, & +1 & -1 & +1 & -1 & 0,555662 \\
7, & -1 & +1 & +1 & +1 & 1,669269 \\
8 & +1 & +1 & & & 1,082872 \\
\hline
\end{tabular}


A matriz de planejamento fatorial foi avaliada através da análise de variância para as variáveis estudados do planejamento fatorial. A Tabela 7 demonstra os principais efeitos dos variáveis espessura de fatia, branqueamento, concentração de $\mathrm{NaCl}$ na desidratação osmótica, bem como temperatura de operação do secador, nas constantes de secagem obtidas pelo ajuste do modelo matemático de secagem Midilli e Kucuk.

Tabela 7 - Análise de variância dos efeitos principais dos fatores estudados.

\begin{tabular}{cccc}
\hline Fator & Quadrado Médio & Teste F & Nível de Significância \\
\hline Espessura & 4,608332 & 111,2648 & $<0,0000001$ \\
Branqueamento & 0,112610 & 2,7189 & 0,127404 \\
Concentração de NaCl & 0,007877 & 0,1902 & 0,671193 \\
Temperatura & 1,246926 & 30,1061 & $<0,000190$ \\
\hline $\mathrm{R}^{2}=92,916 \%$ & & &
\end{tabular}

Os resultados, na Tabela 7, demonstram que a espessura e a temperatura foram significativas na análise de variância ao nível de $95 \%$. Os pré-tratamentos branqueamento e concentração de $\mathrm{NaCl}$ na desidratação osmótica não são significativos para a constante de secagem $(\mathrm{p}=0,05)$. Dandamrongrak et al. (2002) e Borges et al. (2010) verificaram que o pré-tratamento branqueamento, apesar de aumentar a permeabilidade das amostras, não influenciou significativamente nas taxas de secagem, possivelmente devido as elevadas temperaturas de secagem que promoveram a gelatinização do amido de amostras de banana. Segundo Mercali (2009) baixos níveis de desidratação osmótica, como os níveis de 2 e 5\% de cloreto de sódio, geram baixos gradientes de transferência de massa, ou seja, não são capazes de reduzir atividade de água de amostras de banana (Musa sapientum, shum.) a níveis significantes, portanto dificilmente influenciaram nas as taxas de secagem.

A espessura foi o pré-tratamento mais significativo na influência da secagem de banana da variedade terra, seguido da temperatura de operação de secagem, pode-se observar tal resultado através da Figura 5, que apresenta o gráfico de Pareto dos efeitos principais das variáveis de processo na constante de secagem $(\mathrm{K})$.

Resultado semelhante foi encontrado por Madamba et al. (1996), analisando a influencia dos pré-tratamentos na secagem de fatias de alhos, determinaram que os fatores temperatura de secagem e espessura das fatias afetaram diretamente a constante de secagem $(K)$, enquanto a velocidade do fluxo de ar e a umidade relativa não tiveram efeitos significativos. Comportamento semelhante foi encontrado por Silva et al. (2009), ao estudarem a cinética de secagem de fatias de banana variedade maçã em temperaturas de 50,60 e $70^{\circ} \mathrm{C}$. 'Verificaram que o aumento da constante de secagem (K) é em função do decréscimo da espessura e do acréscimo da temperatura. 
Figura 5 - Gráfico de Pareto para a constante de secagem (K)

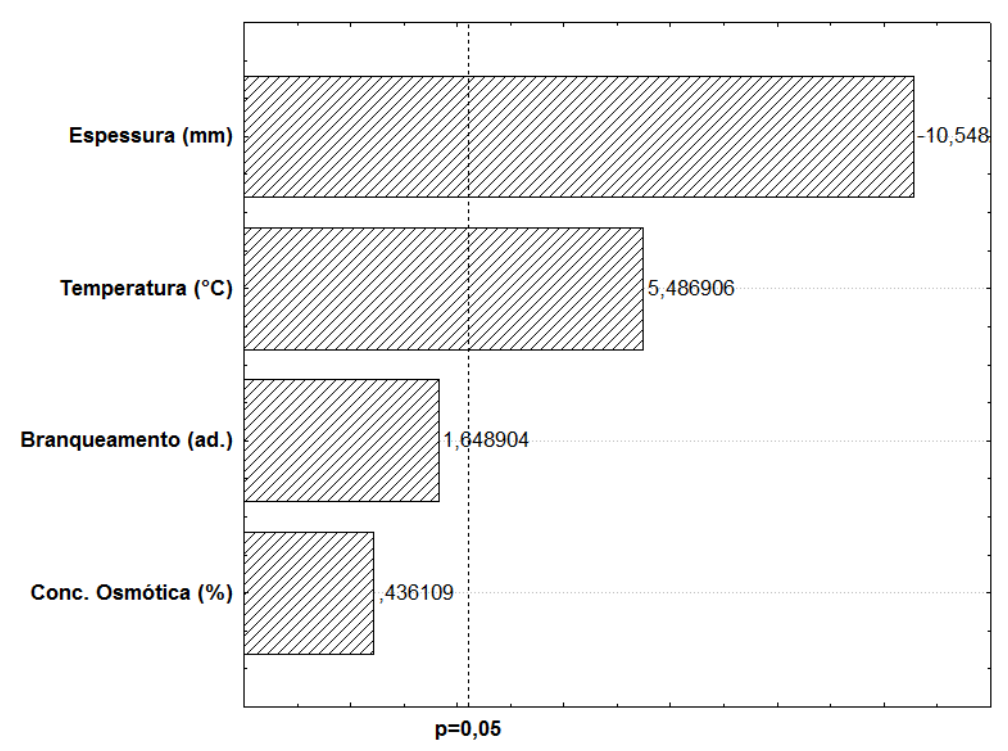

A Figura 6 demonstra os efeitos da espessura da amostra e da temperatura de operação do secador na constante de secagem $(\mathrm{K})$.

Figura 6 - Superfície de resposta para os efeitos da espessura e temperatura na constante de secagem (K).

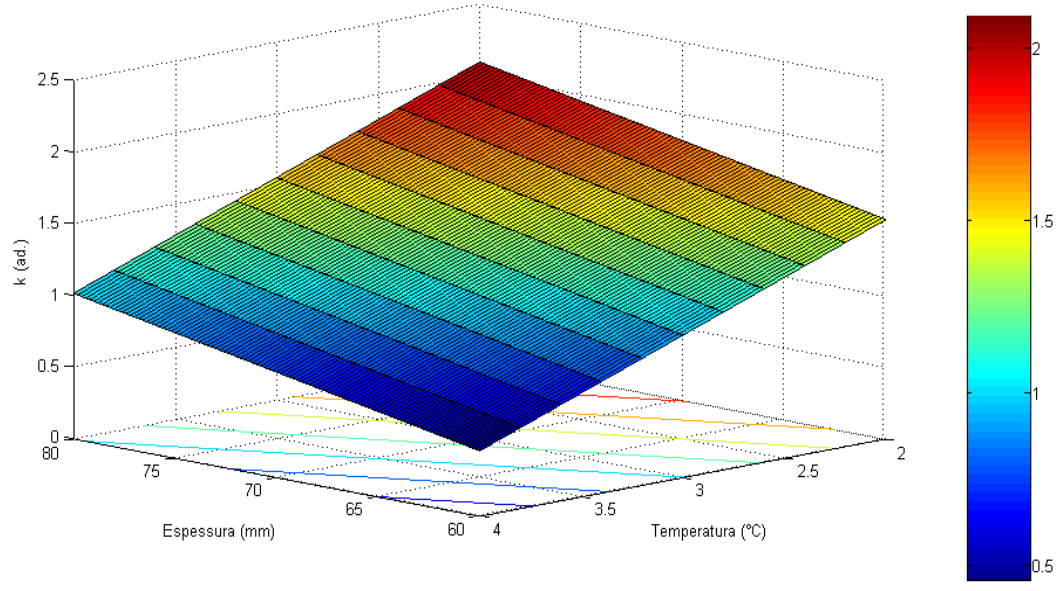

Observa-se na Figura 6 que a constante de secagem (K) foi diretamente proporcional ao aumento da temperatura de operação do secador e inversamente proporcional a espessura das amostras, ou seja, o tempo necessário para as amostras atingirem equilíbrio higroscópico dinâmico foi maior nas menores temperaturas de operação de secagem e nas maiores espessuras das fatias. Segundo Vaccarezza et al.(1974) apud Madamba et al. (1996) verificaram que quanto menor a espessura da fatia maiores são as constantes de secagem devido a menor distância que a umidade deve percorrer até a área superficial exposta de uma determinado volume de produto. Nguyen e Price (2007) observaram um comportamento semelhante ao estudarem a secagem de fatias de 
banana nas espessuras 10 e $20 \mathrm{~mm}$.

Kurozawa (2005) analisou a secagem do cogumelo (Agaricus blazei) com e sem prétratamento osmótico em um secador de leito fixo, através da Metodologia de Superfície de Resposta, encontrou as seguintes condições de secagem otimizadas para maior valor de constante de secagem (K) e menor diferença de cor: $70^{\circ} \mathrm{C}$ e $2,00 \mathrm{~m} / \mathrm{s}$, e $80^{\circ} \mathrm{C}$ e $2,50 \mathrm{~m} / \mathrm{s}$ para o produto sem e com pré-tratamento osmótico, respectivamente;

Porto (2001) avaliou, diante de um planejamento experimental, a influência dos prétratamentos na cinética de secagem de cebola tipo exportação (cv. Baia Periforme) e cebola descarte (classificação miúda) fora do padrão de exportação. A partir das secagens realizadas, expressas na matriz experimental, conclui que as melhores condições de secagem foram alcançadas nos maiores níveis dos fatores estudados, ou seja, cebola (cv. Baia Periforme), temperatura de secagem de $60^{\circ} \mathrm{C}$, branqueada e na forma de pasta.

\section{Conclusão}

O modelo matemático de Midilli e Kucuk foi o que melhor se ajustou aos dados experimentais. Os valores da difusividade efetiva $\left(\mathrm{D}_{\mathrm{EF}}\right)$ para as secagens da matriz de planejamento experimental variaram entre 2,48756 x $10^{-10}$ a $1,02552 \times 10^{-9} \mathrm{~m}^{2} \cdot \mathrm{s}^{-1}$. A espessura das amostras e a temperatura de operação do secador influenciaram significativamente $(p=0,05)$ na constante de secagem, sendo a magnitude da mesma diretamente proporcional ao aumento da temperatura de operação do secador e inversamente proporcional a espessura das amostras. Os pré-tratamentos branqueamento e concentração osmótica não são significativos para a constante de secagem ao nível de significância de 95\%. De acordo com as constantes de secagem apresentadas pelo modelo, recomenda-se a secagem de banana da variedade terra nas seguintes condições: espessura de $2 \mathrm{~mm}$, temperatura de secagem $80^{\circ} \mathrm{C}$ e qualquer outro pré-tratamento, desidratação osmótica com $\mathrm{NaCl}$ e/ou branqueamento.

\footnotetext{
Abstract

This study was conducted to study the influence of different pretreatments on drying of banana variety Terra (Musa sapientum, Linnaeus). The fruits of banana were subjected to three pretreatments: slice thickness ( 2 and $4 \mathrm{~mm}$ ), bleaching (yes and no) and $\mathrm{NaCl}$ concentration ( 2 and $5 \%$ ) in the osmotic dehydration. The drying was performed using a scale of workbench tray dryer. The temperatures of the drying air were 60 and $80^{\circ} \mathrm{C}$ and velocity was approximately to $1 \mathrm{~m} . \mathrm{s}^{-1}$.In order to evaluate and select suitable model for drying, four different empirical models were fitted to experimental data of moisture and compared. To analyze the effects of pretreatments on drying kinetics of banana variety terra, we used a $2_{\text {III }}^{4-1}$ fractional experimental planning, with four factors
} 
and two levels of variation, for the constant drying (K). Among the tested models the model of Midilli and Kucuk had the highest coefficient of determination $\left(\mathrm{R}^{2}\right)$ and the lowest estimated average errors (SE) and is also used to determine the drying constant. With these results, we can conclude: that the mathematical model of Midilli and Kucuk, was the best fitted to the experimental data, the thickness of the samples and the operating temperature of the dryer influenced significantly $(\mathrm{p}=0.05)$ at constant drying, and the magnitude of it was directly proportional to the increase of the operating temperature of the dryer and inversely proportional to the thickness of the samples. According to the constant drying presented by the model, it is recommended to dry banana variety Terra under the following conditions: thickness of $2 \mathrm{~mm}$, drying temperature of $80^{\circ} \mathrm{C}$ and any other pretreatment, osmotic dehydration with $\mathrm{NaCl}$ and/or bleaching.

Key-words: banana variety Terra, drying constant, experimental plannig.

\section{Referências}

AFONSO JÚNIOR, P. C.; CORRÊA, P. C. Comparação de modelos matemáticos para descrição da cinética de secagem em camada fina de sementes de feijão. Revista Brasileira de Engenharia Agrícola e Ambiental, v. 3, n. 03, p. 349-353, 1999.

AKPINAR, E. K.; BICER, Y.; YILDIZ, C. Thin layer drying of red pepper. Journal of Food Engineering, v. 59, n. 01, p. 99-104, 2003. doi:10.1016/S0260-8774(02)00425-9.

ANDRADE, S. A. C; METRI, J. C.; BARROS NETO, B.; GUERRA, N. B. Desidratação osmótica do jenipapo (Genipa americana L.). Revista Ciência e Tecnologia de Alimentos, v. 23, n. 2, p. 276-281, 2003. doi: 10.1590/S0101-20612003000200029.

BABALIS, S. J.; BELESSIOTIS, V. G. Influence of the drying conditions on the drying constants and moisture diffusivity during the thin-layer drying of figs. Journal of Food Engineering, v. 65, n. 3, p. 449-58, 2004. doi:10.1016/j.jfoodeng.2004.02.005.

BORGES, S. V.; MANCINI, M. C.; CORREA, J. L. G.; LEITE, J. Secagem de bananas prata e d'água por convecção forçada. Revista Ciência e Tecnologia de Alimentos, v. 30, n. 3, p. 605-612, 2010.

BROD, F. P. R. Avaliação de um secador vibro-fluidizado. 335f. Tese (Doutorado em Engenharia Agrícola) Faculdade de Engenharia Agrícola -Campinas: Universidade Estadual de Campinas, 2003.

BROOKER, D. B.; BAKKER-ARKEMA, F. W.; HALL, C. W. Drying and storage of grains and oilseeds, Westport. AVI, 1992.

CAMARGO, R. Tecnologia dos Produtos Agropecuários. São Paulo: Nobel, 1986.

CORRÊA, P. C.; ReSEnde, O.; MARTinAZZO, A. P.; GONELI, A. L. D.; BOtelhO, F. M. Modelagem matemática para a descrição do processo de secagem do feijão (phaseolus vulgaris 1.) em camada delgada. Engenharia Agrícola, v.27, n. 02, p.501-507, 2007. doi: 10.1590/S0100-69162007000300020.

DANDAMRONGRAK, R., YOUNG, G.; MASON, R. Evaluation of various pre-treatments for the dehydration of banana and selection of suitable drying models. Journal of Food Engineering, v. 55, n. 2, p. 139-146. 2002. DOI:10.1016/S0260-8774(02)00028-6.

DOYMAZ, I. Air-drying characteristics of tomatoes. Journal of Food Engineering, v. 78, n. 04, p. 1291-1297, 2007a. DOI:10.1016/j.jfoodeng.2005.12.047.

DOYMAZ, I. The kinetics of forced convective air-drying of pumpkin slices. Journal of Food Engineering, v. 79, n. 1, p. 243-248, 2007b. DOI:10.1016/j.jfoodeng.2006.01.049.

DOYMAZ, I. Drying behaviour of green beans. Journal of Food Engineering, v. 69, n. 2, p.161-165, 2005. DOI:10.1016/j.jfoodeng.2004.08.009.

ERTEKIN, C.; YALDIZ, O. Drying of eggplant and selection of a suitable thin layer drying model. Journal of Food Engineering, v. 63, n. 3, p. 349-359, 2004. DOI:10.1016/j.jfoodeng.2003.08.007. 
FELLOWS, P. Tecnología del procesado de los alimentos: Principios y prácticas. Zaragoza (España): Editorial Acribia, S.A., 1994. 549p.

GEANKOPLIS, C. J. Procesos de Transporte y Operaciones Unitarias. 3ed. Mexico: Compañía Editorial Continental, S.A. De C.V. 1998.

GONELI, A. L. D.; CORRÊA, P. C.; JÚNIOR, P. C. A.; OLIVEIRA, G. H. H. Cinética de secagem dos grãos de café descascados em camada delgada. Revista Brasileira de Armazenamento. v. Especial Café, n. 11, p. 74-80, 2009.

GOYAL, R. K.; KINGSLY, A. R. P.; MANIKANTAN, M. R.; ILYAS, S. M. Thin-layer Drying Kinetics of Raw Mango Slices. Biosystems Engineering. v. 95, n. 1, p. 43-49, 2006. DOI:10.1016/j.biosystemseng.2006.05.001.

GURJÃO, K. C. de O. Desenvolvimento, armazenamento e secagem de tamarindo (Tamarindus Indica L.). Tese (Doutorado em Agronomia) -Areia, Universidade Federal da Paraíba, 2006.

JESUS, C.; FOLEGATTI, M. I. S.; MATSUURA, F. C. A. U. ; CARDOSO, R. L. Caracterização física e química de frutos de diferentes genótipos de bananeira. Bragantia, Campinas, v. 63, n. 3, p. 315-323, 2004. DOI: 10.1590/S000687052004000300001 .

KALEEMULLAH S.; KAILAPPAN R. Modelling of thin-layer drying kinetics of red chillies, Journal of Food Engineering, v. 76, n. 4, p. 531-537, 2006. DOI:10.1016/j.jfoodeng.2005.05.049.

KUROZAWA, L. E. Efeitos das condições de processo na cinética de secagem de cogumelo (Agaricus blazei). Dissertação de Mestrado, Campinas, UNICAMP, 2005.

LAHSASNI, S.; KOUHILA, M.; MAHROUZ, M.; JAOUHARI, J.T. Drying kinetcs of prickly pear fruit (Opuntia ficus indica). Journal of Food Engineering, v. 61, n .2, p. 173-179, 2004. DOI:10.1016/S0260-8774(03)00084-0.

MADAMBA, P.S.; DRISCOLL, R.H.; BUCKLE, K.A. Thin-layer drying characteristcs of garlic slices. Journal of Food Engineering, v. 29, n. 1, p. 75-97, 1996. DOI:10.1016/0260-8774(95)00062-3.

MARQUES, L. F., DUARTE, M. E. M., MATA, M. E. R. M. C., NUNES, L. S., COSTA, T. L., COSTA, P. B. S., DUARTE, S. T. G. Secagem precedida de desidratação osmótica de pseudofruto de caju: comparação entre modelos matemáticos aplicados. Revista Brasileira de Produtos Agroindustriais, Campina Grande, v. 9, n. 2, p. 161-170, 2007.

MATTHIESEN, M. L. e BOTEON, M. Análise dos principais pólos produtores de banana no Brasil. Revista Hortifuti Brasil, v. 02, n. 16, p. 18, 2003.

MERCALI, G. D. Estudo da transferência de massa na desidratação osmótica de banana (Musa sapientum, shum.). Dissertação de Mestrado, UFRGS, Porto Alegre, RS. 2009.

MENGES, H. O.; ERTEKIN, C. Mathematical modeling of thin layer drying of Golden apples. Journal of Food Engineering, v. 77, n. 1, p. 119-125, 2006. DOI:10.1016/j.jfoodeng.2005.06.049.

MIDILLI, A.; KUCUK, H.; YAPAR, Z. A. New model for single-layer drying. Drying Technology, v.20, p. 15031513, 2002. DOI:10.1081/DRT-120005864.

NGUYEN, M. H.; PRICE, W. E. Air-drying of banana: influence of experimental parameters, slab thickness, banana maturity and harvesting season. Journal of Food Engineering, v. 79, n. 1, p. 200-207, 2007. doi:10.1016/j.jfoodeng.2006.01.063.

OZDEMIR, M.; DEVRES, Y.O. The thin layer drying characteristics of hazelnuts during roasting. Journal of Food Engineering, v. 42, n. 04, p. 225-233, 1999. DOI: 10.1016/S0260-8774(99)00126-0.

PANCHARIYA, P.C.; POPOVIC, D.; SHARMA, A.L. Thin-layer modeling of black tea drying process. Journal of Food Engineering, v. 52, v. 04, p. 349-357, 2002. DOI: 10.1016/S0260-8774(01)00126-1.

PARK, K. J.; YADO, M. K. M.; BROD, F. P. R. Estudo de secagem de pêra bartlett (pyrus sp.) em fatias. Ciência e Tecnologia de Alimentos, v. 21, n. 03, p. 288-292, 2001. DOIi:10.1590/S0101-20612001000300007.

PONTES, S. F.; BONOMO O. R. C. F.; PONTES, L. V.; RIBEIRO, A. C.; CARNEIRO, J. C. S. Secagem e avaliação sensorial de banana da terra. Revista Brasileira de Produtos Agroindustriais, v. 9, n. 2, p. 143-148, 2007. 
PORTO, P. S. S. Cinética da secagem de cebola (Allium cepa L.) em camada delgada. Dissertação de mestrado, FURG, Rio Grande, RS. 2001.

RODRIGUES, M. I e IEMMA, A. F. Planejamento de experimentos e otimização de processos - uma estratégia sequencial de planejamentos. São Paulo: Casa do Pão, 2005.

SACILIK K.; KONURALP A. E. The thin layer drying characteristics of organic apple slices, Journal of Food Engineering, v. 73, n. 03, p. 281-289, 2006. doi:10.1016/j.jfoodeng.2005.03.024.

SARAVACOS, G.; ROUZEOUS, G.S. Diffusivity of moisture in air-drying of starch gels. Engineering and Food, London and New York: Brian M. Mckenna, Elsevier Applied Science Publishes, v.1, p. 493-507, 1984.

SILVA, A. S.; MELO, K. S.; ALVES, N. M. C.; FERNANDES, T. K. S.; FARIAS, P. A. Cinética de secagem em camada fina da banana maçã em secador de leito fixo. Revista Brasileira de Produtos Agroindustriais, v. 11, n. 2, p. 129-136, 2009.

SILVA, A. S., ALMEIDA, F. de A. C., SILVA, F. L. H. da, DANTAS, H. J., LIMA, E. E. Desidratação e efeito de prétratamentos no extrato seco do pimentão verde. Revista Brasileira de Produtos Agroindustriais, v. 10, n. 1, p. 27-34, 2008.

SILVA, F. D., MATA, M. E. E. M. C., DUARTE, M. E. M., SOUZA, J. A., SILVA, Y. C. e. Desidratação osmótica de banana da terra (Musa sapientum) aplicação de modelos matemáticos. Revista Brasileira de Produtos Agroindustriais, v. esp., n. 1, p. 69-76, 2003.

STRUMILO, C. e KUDRA, T. - Drying: Principles, Applications and Design. In: Hughes, R. Topics in Chemical Engineering. v. 3, Universidade de Salford, UK. 1986

SOUZA NETO, M. A.; MAIA, G. A.; LIMA, J. R.; FIGUEIREDO, R. W.; SOUZA FILHO, M. S. M.; LIMA, A. S. Desidratação osmótica de manga seguida de secagem convencional: avaliação das variáveis de processo. Ciência Agrotecnologia, v. 29, n. 5, p. 1021-1028, 2005.

VASQUES, A. R.; VALLE, R. C. S. C.; VALLE, J. A. B. Estabelecimento de parâmetros de desidratação de fatias de maçã fuji e gala com estudo de preferência sensorial. In: Congresso Brasileiro de Engenharia Química, Curitiba, 2004.

WANG, Z.; SUN, J.; LIAO, X.; CHEN, F.; ZHAO, G.; WU, J.; HU, X. Mathematical modeling on hot air drying of thin layer apple pomace. Food Research International, v. 40, n. 01, p. 39-46, 2007. doi:10.1016/j.foodres.2006.07.017.

ZOGZAS, N. P.; MAUROULIS, Z. B.; MARINOS-KOURIS, D. Moisture diffusivity data compilation in foodstuffs. Drying Technology, v. 14, n. 10, p. 2225-2253, 1996. DOI:10.1080/07373939608917205

\section{Dados dos autores:}

Nome completo: Philipe dos Santos

Filiação institucional: Universidade do Estado de Mato Grosso (UNEMAT) - Campus Universitário "Dep. Est. Renê Barbour"

Departamento:Engenharia de Alimentos

Função ou cargo ocupado: Discente do Curso de Engenharia de Alimentos

Endereço: Rua A, s/n, Bairro São Ramuindo, Barra do Bugres-MT, Brasil 78390-000

Telefones para contato:(66) 9984-5169

e-mail:ph_sinop@hotmail.com

Nome completo: Fabricio Schwanz da Silva

Filiação institucional: Universidade do Estado de Mato Grosso (UNEMAT) - Campus Universitário "Dep. Est. Renê Barbour"

Departamento:Engenharia de Produção Agroindustrial 
Função ou cargo ocupado: Docente do Curso de Engenharia de Produção Agroindustrial

Titulação: Doutor em Engenharia Agrícola

Endereço: Rua A, s/n, Bairro São Ramuindo, Barra do Bugres-MT, Brasil 78390-000

Telefones para contato:(65) 3361-1413

e-mail:fabricio@unemat.br

Nome completo: Alexandre Gonçalvez Porto

Filiação institucional: Universidade do Estado de Mato Grosso (UNEMAT) - Campus

Universitário "Dep. Est. Renê Barbour"

Departamento:Engenharia de Alimentos

Função ou cargo ocupado: Docente do Curso de Engenharia de Alimentos

Titulação: Doutor em Tecnologia de Sementes

Endereço: Rua A, s/n, Bairro São Ramuindo, Barra do Bugres-MT, Brasil 78390-000

Telefones para contato:(65) 3361-1413

e-mail: agporto@unemat.br

Nome completo: Flávio Teles de Carvalho

Filiação institucional: Universidade do Estado de Mato Grosso (UNEMAT) - Campus

Universitário "Dep. Est. Renê Barbour"

Departamento:Licenciatura em Matemática

Função ou cargo ocupado: Docente do Curso de Licenciatura em Matemática

Titulação: Doutor em Física Aplicada

Endereço: Rua A, s/n, Bairro São Ramuindo, Barra do Bugres-MT, Brasil 78390-000

Telefones para contato:(65) 3361-1413

e-mail: ftcsilva@gmail.com

Nome completo: Guilherme de Figueiredo Furtado

Filiação institucional: Universidade do Estado de Mato Grosso (UNEMAT) - Campus

Universitário "Dep. Est. Renê Barbour"

Departamento:Engenharia de Alimentos

Função ou cargo ocupado: Discente do Curso de Engenharia de Alimentos

Endereço completo para correspondência

Rua A, s/n, Bairro São Ramuindo, Barra do Bugres-MT, Brasil 78390-000

Telefones para contato:(65) 3361-1413

e-mail: guilherme.sartori@hotmail.com 